\title{
Adrenal Chromaffin Cell Co-Grafts With NGF-Secreting Fibroblasts
}

\author{
Paul H. Patterson ${ }^{1}$, Kenji Niijima ${ }^{1}$, Gordon R. Chalmers ${ }^{2}$, Daniel A. Peterson ${ }^{2}$, \\ Lisa J. Fisher ${ }^{2}$ and Fred H. Gage ${ }^{2}$ \\ ${ }^{1}$ Biology Division, California Institute of Technology, Pasadena, CA 91125, and ${ }^{2}$ Department of \\ Neurosciences, University of California at San Diego School of Medicine, La Jolla, CA 92093-0627, USA
}

Autografts offer distinct advantages over neural grafts of non-self origin. While adrenal chromaffin cells (ACC) have been used extensively in grafts to the CNS, their potential for neuronal differentiation has been underutilized. ACC are mitotically active, multipotential neuronal progenitors that respond to instructive differentiation signals by adopting any of several neuronal phenotypes. For instance, these cells are potential sources for cholinergic as well as catecholaminergic neuronal grafts (Mahanthappa, Gage and Patterson, Prog Brain Res 1990; 82: 33-39).

One approach for enhancing ACC survival and neurite outgrowth in the CNS is to provide a source of nerve growth factor (NGF). We find that skin fibroblasts genetically engineered to secrete high levels of NGF (Kawaja and Gage, Neuron 1991; 7: 1019-1030) promote such neuronal differentiation by perinatal rat $\mathrm{ACC}$ when cografted into the striatum of adult rats. These ACC exhibit greatly enlarged soma size, extensive process outgrowth, and are positive for tyrosine hydroxylase, neurofilament (NF200), NGF receptor (p75), as well as the neuronspecific markers SCG10 and MAP2. The effect of NGF secretion on graft volume and the total number of ACC, as defined by morphology with Nissl and tyrosine hydroxylase staining, was determined using unbiased stereology. The number of ACC surviving at 8 weeks was minimal without co-grafted fibroblasts, and ACC survival with the NGF-secreting fibroblasts was enhanced 2.5-fold over the co-grafting with control fibroblasts. The ability of control fibroblasts to enhance survival dramatically without inducing neuronal differentiation merits further investigation. The NGF transgene induces virtually all $\mathrm{TH}^{+}$cells to express the neuronal markers SCG10 and MAP2. Neurite outgrowth by these cells was largely confined to within the graft, however. The survival rate (a mean of $42 \%$ of initial ACC grafted) and differentiation (virtually all $\mathrm{TH}^{+}$cells) observed in this co-graft paradigm are the most significant yet reported for adrenal grafts.

The effects of these long-term grafts on the animals' behavior in Parkinson's disease paradigms are currently being tested. 

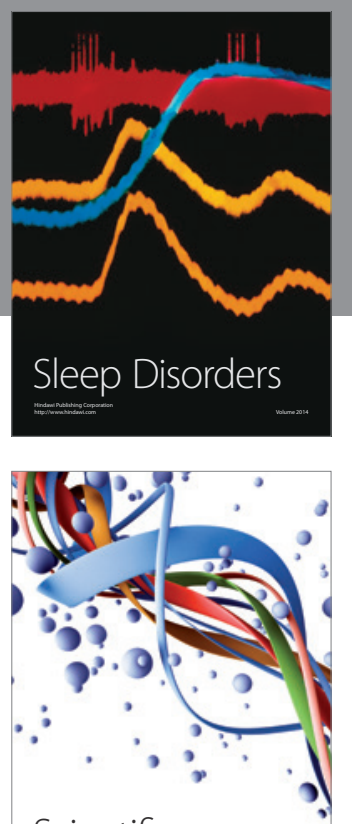

Scientifica
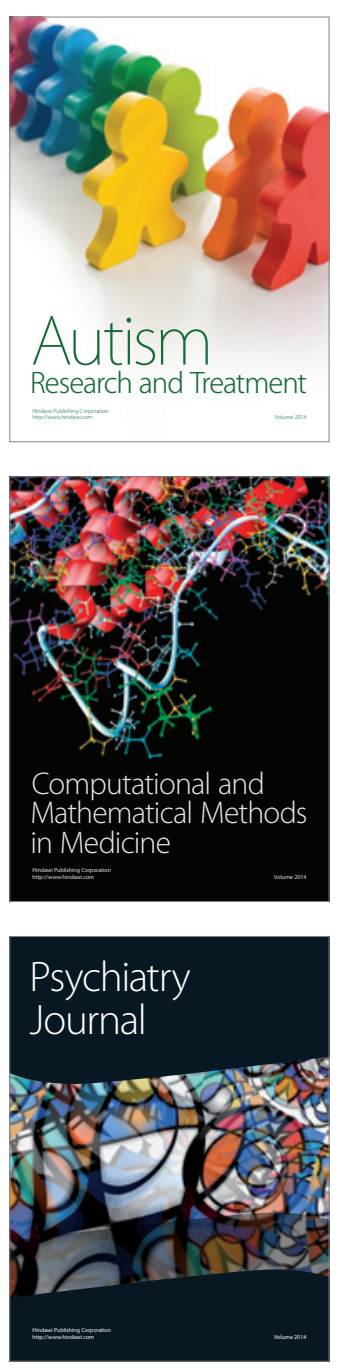
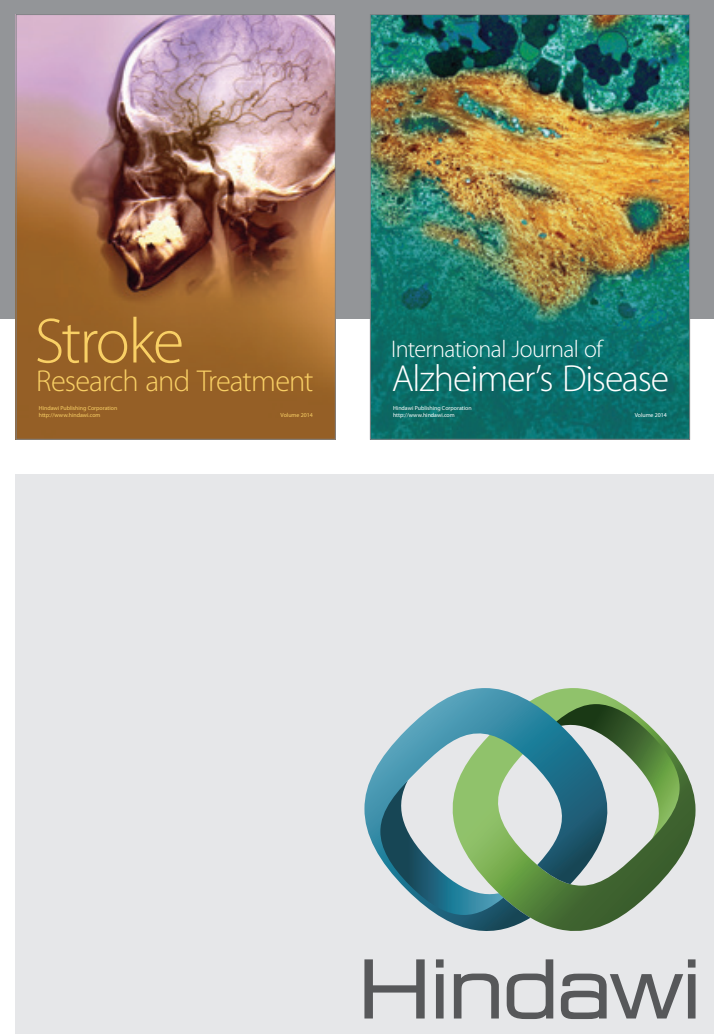

Submit your manuscripts at

http://www.hindawi.com
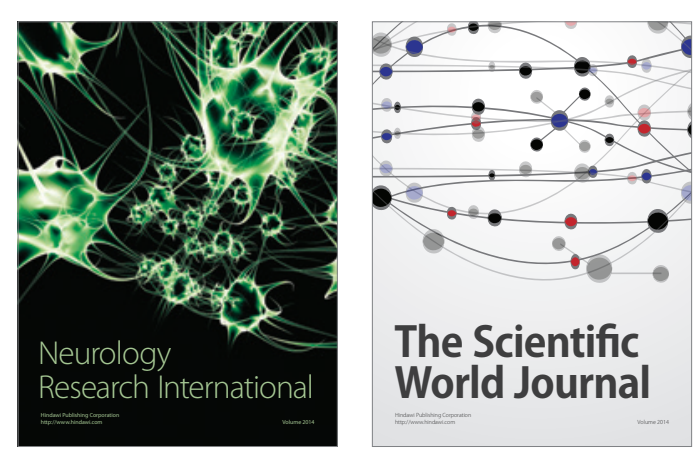

The Scientific World Journal

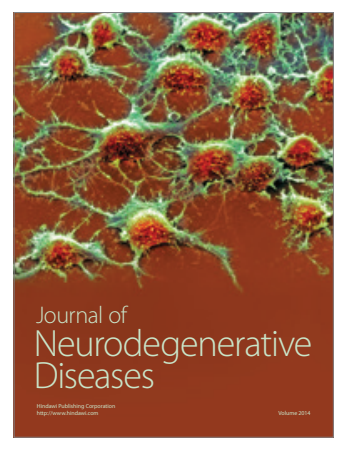

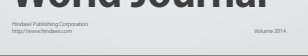

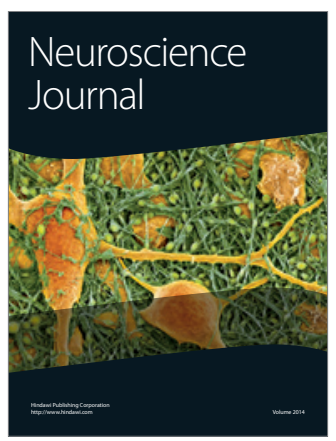

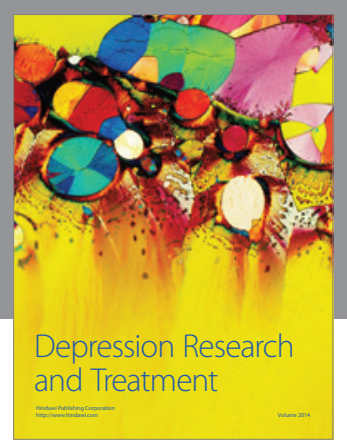
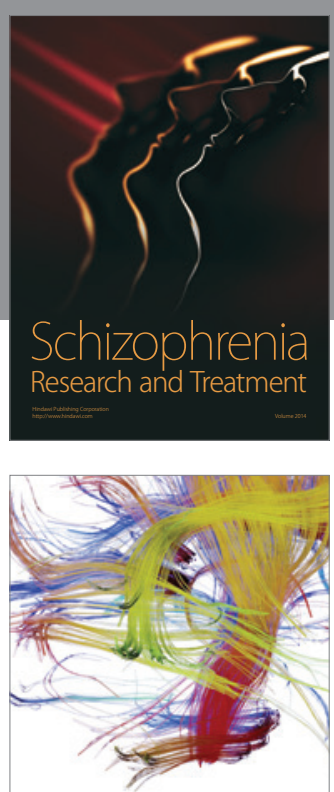

Brain Science

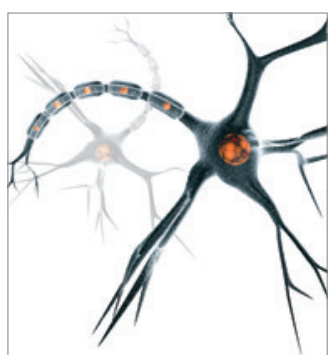

Neural Plasticity
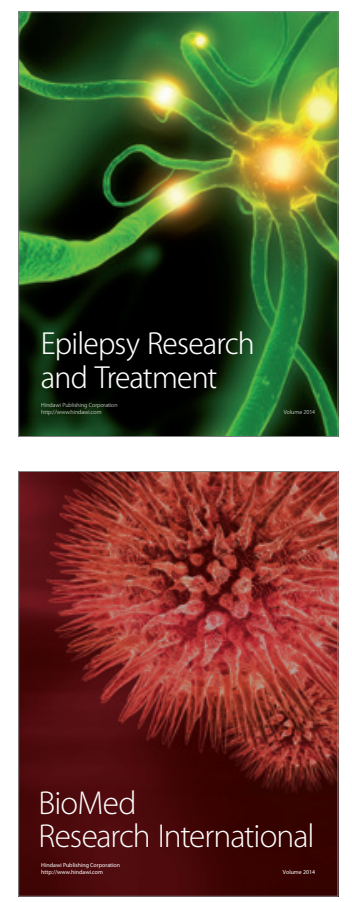

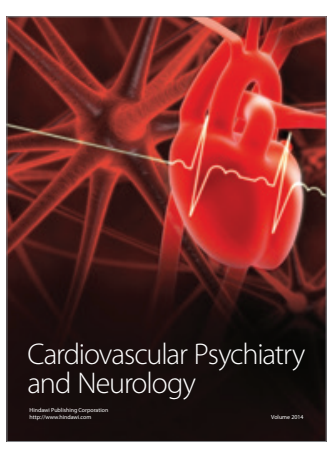

Parkinson's

Disease
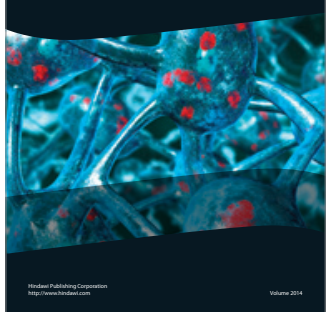